\title{
Building a LLNL Capability in Radioactive Ion Beam Experiments
}

L.A. Bernstein, J.A. Becker, P.E. Garrett, W. Younes, A. Schiller

\section{January 31, 2002}

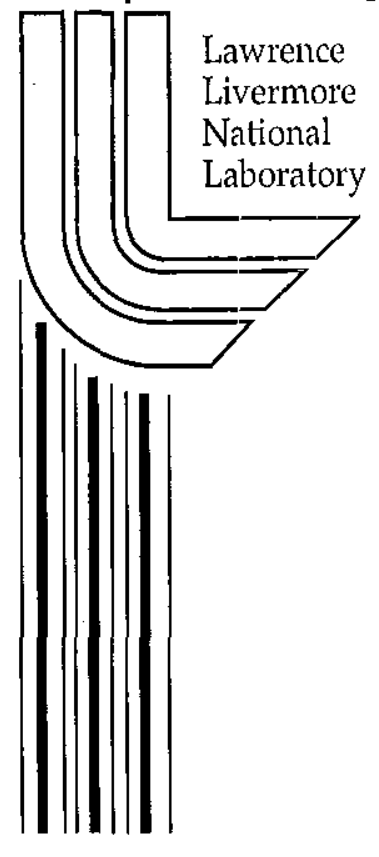




\section{DISCLAIMER}

This document was prepared as an account of work sponsored by an agency of the United States Government. Neither the United States Government nor the University of California nor any of their employees, makes any warranty, express or implied, or assumes any legal liability or responsibility for the accuracy, completeness, or usefulness of any information, apparatus, product, or process disclosed, or represents that its use would not infringe privately owned rights. Reference herein to any specific commercial product, process, or service by trade name, trademark, manufacturer, or otherwise, does not necessarily constitute or imply its endorsement, recommendation, or favoring by the United States Government or the University of California. The views and opinions of authors expressed herein do not necessarily state or reflect those of the United States Government or the University of California, and shall not be used for advertising or product endorsement purposes.

This work was performed under the auspices of the U. S. Department of Energy by the University of California, Lawrence Livermore National Laboratory under Contract No. W-7405-Eng-48.

This report has been reproduced directly from the best available copy.

Available electronically at http:L/www.doc.gov/bridge

Available for a processing fee to U.S. Department of Energy

And its contractors in paper from

U.S. Department of Energy

Office of Scientific and Technical Information

P.O. Box 62

Oak Ridge, TN 37831-0062

Telephone: (865) 576-8401

Facsimile: (865) 576-5728

E-mail: reports@adonis.osti.gov

Available for the sale to the public from

U.S. Department of Commerce

National Technical Information Service

5285 Port Royal Road

Springfield, VA 22161

Telephone: (800) 553-6847

Facsimile: (703) 605-6900

E-mail: orders@ntis.fedworld.gov

Online ordering: http://www.ntis.gov/ordering.htm

OR

Lawrence Livermore National Laboratory

Technical Information Department's Digital Library

http://www.llnl.gov/tid/Library.html 


\title{
Building a LLNL Capability in Radioactive Ion Beam Experiments
}

\author{
L.A. Bernstein \\ Principal Investigator \\ J.A. Becker, P.E. Garrett, W. Younes, A. Schiller \\ Co-investigators \\ i \\ Proposal Tracking Number: 00-ERD-035
}

\begin{abstract}
$\underline{\text { Abstract }}$
The purpose of this LDRD was to establish a program at LLNL in radioactive ion beam (RIB) experiments that would use these experiments to address a wide range of physics issues in both stellar nucleosynthesis and stockpile stewardship radiochemistry. The LDRD was funded for a total of two years (fiscal years 2000 and 2001) and transferred to the Physical Data Research Program in físcal year 2002.

Reactions on unstable nuclei and isomeric states play a central role in the formation of elements in both stars and nuclear devices. However, the abilities of reaction models to predict cross sections on radioactive nuclei are uncertain at best. This can be attributed to the lack of experimental data to guide reaction-modeling efforts. Only the $10 \%$ of all bound nuclei that can be formed with stable targets and beams have been accessed and studied. The proposed Rare Isotope Accelerator (RIA) and existing RIB facilities offer an unprecedented opportunity to address many of the outstanding questions in nuclear structure, reactions and astrophysics by enabling the observation of nuclear reactions with radioactive targets and/or beams. The primary goal of this LDRD is to develop three experimental capabilities for use with RIB experiments:
\end{abstract}

- Level density and $\gamma$-ray strength function measurements using statistical $\gamma$-rays.

- Charged particle-induced cross sections measurements on radioactive nuclei.

- Neutron-induced cross section measurements on a radioactive target.

RIA and RIB based experiments are the new frontier for nuclear physics. The joint DOE/NSF nuclear science advisory committee has named development of a RIA facility in the United States as the highest new construction priority. In addition to addressing the questions presented above, this LDRD has helped to establish a position for LLNL at the forefront of the international nuclear science community 


\section{Table of Contents}

1. Scientific Motivation

1.1. How elements are made in stars and nuclear devices.

1.2. The importance of RIB experiments

2. Accomplishments

2.1. ${ }^{235} \mathrm{U}(\mathrm{n}, \mathrm{f})$ measurements for RIB Ion Source development

2.2. Oslo Level Density Measurements

2.3. The Yale Experiment

2.4.The Development of "Re-cyclotron" Radioactive Beams at LBNL. 2.5. Development of a Highly Segmented Particle Detector (HSPD) for RIB experiments.

2.6.The ANL Secondary Beam Experiment

2.7.RIBS and Stockpile Stewardship Workshop at LLNL

3. Summary of Deliverables Future Plans

\section{1) Scientific Motivation}

\section{1) How elements are made in stars and nuclear devices}

All elements with atomic number greater than 4 (Boron) are formed in stellar interiors through a process called stellar nucleosynthesis. Stellar nucleosynthesis can be broadly divided into explosive (including supernovae, novae and other exotic cosmological events) and non-explosive (including processes currently taking place in the sun) mechanisms. Explosive nucleosynthesis takes place in high-energy and particle density environments where most nuclear reactions occur on unstable nuclei prior to their decay. However, even in non-explosive nucleosynthesis the few reactions that occur on a radioactive nucleus play an especially important role in determining the abundance of the elements created.

Three processes account for the majority of nuclei formed in explosive nucleosynthesis. The main mechanisms are referred to as the $\mathrm{r}$ - (for rapid neutron capture) and the rp- (rapid proton capture) processes. However, 32 of the lightest stable isotopes from ${ }^{74} \mathrm{Se}(\mathrm{Z}=34)$ to ${ }^{196} \mathrm{Hg}(\mathrm{Z}=80)$, referred to as "p-nuclei", are not formed in either of these processes. Two models have been suggested for their production [Sau97]. The first of these consists of a series of $(\gamma, n)$ reactions followed by $\beta$-decay of the products back to stability. The second process involves $(\gamma, p)$ and $(\gamma, \alpha)$ reactions in a neutrino-driven wind. Nuclear reactions on radioactive nuclei and isomeric states play a critical role in both of these models of [Woo94,Hof96]. Both models are hampered by a near complete lack of experimental information of the relevant cross sections [Bao00]. The current approach involves using statistical model reaction codes to calculate the relevant cross sections. However, these codes have recently been shown to lack quantitative predictive capability [Ber97,Gar00]. A particular weakness in the models is a lack of knowledge of two important properties that influence the calculated cross section: the level density and the $\gamma$-ray strength function.

The main non-explosive nucleosynthesis mechanism which produces elements from Iron $(Z=26)$ to Bismuth ( $Z=83$ ) is referred to as the $s$ - (or slow) process. In the s-process neutrons are captured on stable nuclei until the capture rate on the radioactive nucleus formed is comparable to its decay rate. At this point the s-process "branches" into two paths. These branch points can provide unique insight into the stellar environment where the s-process takes place. Unfortunately, the same weaknesses in the explosive 
nucleosynthesis reaction models are present here as well. Only a direct measurement of neutron capture cross sections on the radioactive branch point nuclei, or the use of surrogate charged particle reactions, such as $(D, p)$, would eliminate the uncertainty in sprocess modeling.

The interpretation of Stockpile Stewardship radiochemical data shares many similarities with stellar nucleosynthesis models in that both involve reactions on largely radioactive nuclei and isomeric nuclear states. As a result radiochemistry suffers from the same lack of nuclear reaction information. A recent survey of nuclear data used in radiochemical studies [Net99] shows that with the exception of single $(n, 2 n)$ measurement in ${ }^{88} \mathrm{Zr}$, none of the four "most reliable" radiochemical data sets, Zirconium, Yttrium, Lutetium and Bismuth, contain any measurements on radioactive nuclei. Furthermore, even though half of the cross sections in the radiochemical network calculations are on isomeric states, no cross section measurements on an isomer have ever been performed. Figure 1 (at the end of section 1.2) shows a portion of the chart of the nuclides between Bromine $(Z=35)$ and Zirconium $(Z=40)$. Nuclei of interest to Stockpile Stewardship radiochemistry are marked in red. The dashed and solid arrows show the p-process pathway. S-process branch point nuclei are marked in blue. In many cases the same reaction that is important for nuclear astrophysics is also important to radiochemistry. This LDRD will exploit this synergy to perform measurements that are simultaneously valuable to both the astrophysical and radiochemical communities.

\section{2) How RIBS can help}

The most obvious way that RIB experiments can be used to improve nucleosynthesis and radiochemical interpretation models is through direct measurement of cross sections on both radioactive nuclei and isomeric states. A measured cross section can be used not only in a reaction network, but also to guide improvements to reaction models.

The cross sections of interest can be grouped into neutron-induced and charged particle-induced reactions. Neutron induced reaction, including most notably $(\mathrm{n}, \gamma)$ reactions, are of the most direct value since they make up the majority of the reactions in stellar and radiochemical reaction networks. However, measurement of neutron-induced cross section on radioactive nuclei are notoriously more difficult than charged-particle measurements. This is due to the fact that they require the formation of a radioactive target since a pure neutron target does not exist. An alternative to this is to produce radioactive targets and use a very intense ( $>10^{\circ}$ neutrons/second) neutron source. Prestwood et al., [Pre84] performed one of these measurements using an ${ }^{88} \mathrm{Y}\left(\mathrm{t}_{1 / 2}=106.65\right.$ days) target and the world's most intense $14 \mathrm{MeV}$ neutron source $\left(6 \times 10^{12}\right.$

neutrons/second into $4 \pi$ ). However, no work has been done on this front recently. Some initial efforts have been made under this LDRD to develop long-lived radioactive targets $\left(t_{1 / 2}>10\right.$ days) for neutron-induced cross section measurements, but not definitive plans have been made to date.

Some charged particle reactions, including (D,p), can be used as surrogates for $(n, \gamma)$ measurements when the direct neutron measurement is not experimentally feasible due to the short life of the nucleus involved. The translation of $(D, p)$ cross section information into $(n, \gamma)$ requires a detailed theoretical calculation that is beyond the scope of this proposal to discuss. These experiments involve the use of "inverse kinematics" with a deuterated polyethylene target. Inverse kinematics involves accelerating a heavier-mass projectile onto a lighter-mass target. This approach has two inherent advantages over the use of a radioactive target. Firstly, the amount of background radioactivity at the experiment site is smaller. Secondly, it allows for measurements of short-lived radioactive species where the assembly of a radioactive target is impossible. Efforts are 
underway to measure $(\mathrm{D}, \mathrm{p})$ reaction on ${ }^{76} \mathrm{Kr}\left(\mathrm{t}_{1 / 2}=14.1\right.$ hours $),{ }^{92 \mathrm{~m}} \mathrm{Nb}\left(\mathrm{t}_{\mathrm{I} / 2}=10.15\right.$ hours $)$ and ${ }^{92 \mathrm{~g}} \mathrm{Nb}\left(\mathrm{t}_{1 / 2}=34.7\right.$ million years) using the 88-Inch Cyclotron at Lawrence Berkeley National Laboratory. This work is described in section 2.6 below.

We have also explored another technique for measuring charged particle induced cross sections; secondary beams. In a secondary beam experiment a radioactive nucleus is formed using a heavy-ion reaction, often in inverse kinematics. This radioactive nucleus is then separated from the beam using an electromagnetic recoil separator. The resulting "secondary beam:" is then focused onto a target. We have performed a secondary beam experiment at Argonne National Laboratory in February 2001. We plan of proposing another experiment of this sort in collaboration with the Michigan State University Group in 2002. This effort is described in section 2.3 below.

Other improvements can be made to stellar nucleosynthesis and radiochemical nuclear reaction models in addition to cross section measurements. Most nuclear reaction models used to generate stellar nucleosynthesis and radiochemical reaction networks are statistical Hauser-Feshbach theory [Hau52]. These calculations are highly dependent on the density of states in the nucleus, also referred to as the level density, as a function of excitation energy and spin. Since nuclear level densities are highly dependent on nuclear structure, it is necessary to measure them in the nuclei of interest. At low energies $\left(\mathrm{E}_{\mathrm{x}}<2-\right.$ $3 \mathrm{MeV}$ ) this can be accomplished using the techniques of discrete $\gamma$-ray and particle spectroscopy. However, since the level density in the nucleus rises rapidly with excitation energy, especially above the "pair" gap of $1.2-2.4 \mathrm{MeV}$, a technique needs to be developed to measure level densities above this energy.

In order to do address this need we are adapting an approach pioneered at the University of Oslo for use with RIB experiments. The approach measures level densities as a function of excitation energy using statistical $\gamma$-ray spectroscopy [Gut87,Tve96]. Our intention is to adapt the technique for use in inverse kinematics RIB experiments. This involves increasing the efficiency of the detectors used by a factor of 100 in order to accommodate the relatively weak beam currents typical of RIB experiments.

In addition to making this technique compatible with RIB experiment, we will add the ability to measure the dependence of the level density on spin through coupling the technique to the world's state of the art $\gamma$-ray spectrometer, GAMMASPHERE. The Oslo experiments involved only the use of low-resolution $\gamma$-ray spectrometers. This did not allow for the observation of individual discrete $\gamma$-rays from states of known spin. In our GAMMASPHERE experiments we will not only record statistical $\gamma$-ray spectra using low-resolution $\gamma$-ray detectors, but also add the capabilities of the high-resolution Ge detectors. This part of the program has been the principal focus of our efforts to date and is described in section 2.1 below. 
Figure 1: A region of the chart of the nuclides where from Selenium to Zirconium showing the overlap in nuclei used for radiochemical diagnostics, and s- and p-process radiochemical network calculations. Also note that most of these nuclei contain isomers that need to taken into account in both radiochemical and astrophysical models.

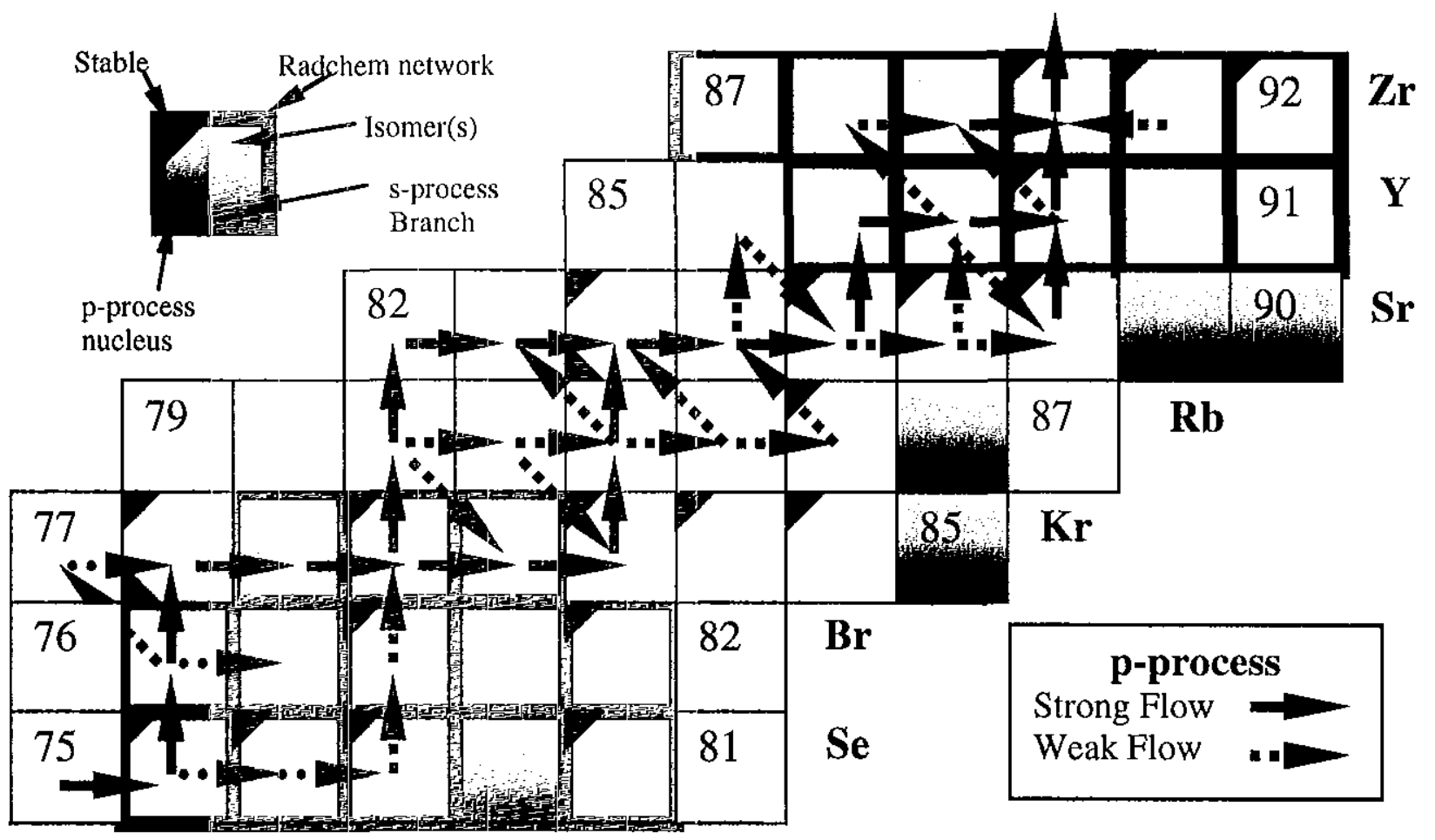




\section{2) Accomplishments and Technical Descriptions}

In this section we will describe our accomplishments and provide a technical description of the methods and facilities used.

\section{$\underline{2.1}{ }^{235} \mathrm{U}\left(\mathrm{n}_{2} \mathbf{f}\right)$ studies for RIB target design}

Neutron-rich radioactive beams provide a wealth of information for nuclear astrophysics, structure and reaction models. However, these nuclei are particularly hard to produce since nuclear fusion reactions result in the formation of nuclei on the neutron-deficient side of the stability. Only neutron capture and fission produce nuclei with a surplus of neutrons. However, even the neutron fluxes present in a high-flux reactor $\left(10^{15} \mathrm{n} / \mathrm{s} / \mathrm{cm}^{2}\right)$ are only sufficient to allow for the first 1 or 2 sequential neutron capture reactions before encountering a nucleus that will $\beta$-decay before capturing an additional neutron.

This leaves fission as the preferred mechanism for forming neutron-rich nuclei. One common technique employed is to use neutron-induced fission on an Actinide (most often Uranium) target that is part of a high-voltage ion source. This technique is referred to as and Isotope Separator On-Line (ISOL) ion source. Recent ISOL designs have emphasized the use of so called "secondary" neutrons that are produced by spallation of an intense proton beam to initiate fission on the ISOL Actinide target. However, these target designs are hampered by a lack of fragment production cross sections for highenergy $(10+\mathrm{MeV})$ neutron-induced fission.

This LDRD has played a role in determining production cross sections for neutroninduced fission on ${ }^{235} \mathrm{U}$ for neutron energies from $600 \mathrm{keV}$ to $250 \mathrm{MeV}$. This data was obtained using the GEANIE (GErmanium Array for Neutron Induced Excitations) spectrometer at Los Alamos National Laboratory. This work has been carried out by Dr. Walid Younes of LLNL and is the topic of a Physical Review C article published in December 2001 [You01]. Some of the results from this article are shown in figure 2. 


$$
\frac{11}{\Delta L}
$$




\section{2) Level Density Measurements at the University of Oslo}

The level density measurement technique developed at the CACTUS array at the University of Oslo (see figure 3 below) involves a coincident measurement of the outgoing particle and $\gamma$-ray cascade following the $\left({ }^{3} \mathrm{He}, \alpha \gamma\right)$ transfer reaction. Measuring the outgoing $\alpha$-particle energy allows for determination of the excitation energy of the nucleus when it emitted the $\gamma$-ray cascade. The "first generation" of $\gamma$-rays in this cascade is theoretically given by the product of the level density and the $\gamma$-ray strength function:

$$
\Gamma\left(E_{x}, E_{\gamma}\right)=\rho\left(E_{x}\right) \cdot F\left(E_{\gamma}\right)
$$

The first generation spectrum $\gamma$-ray spectra is obtained experimentally by subtracting $\gamma$-ray cascades resulting from the population of lower excitations from those corresponding to higher excitation energy. This is possible because the transfer reaction populates the reaction product nucleus over a wide range of energies. Repeating this process down to the lowest energies yields a set of "first generation" $\gamma$-ray spectra.

An example of the data is shown in figure 3 below. The level density and $\gamma$-ray strength function are then simultaneously extracted by assuming functional forms for them, and performing a $\chi^{2}$-minimization to fit the data. The reliability of this technique is most readily seen in recent results from Japan [Miz99] where the $\gamma$-ray spectrum following neutron capture on ${ }^{161-163}$ Dy was reproduced by the Oslo experiment leading to the same nucleus [VoiOl].

In December 2000 two experiments were performed at the University of Oslo cyclotron to measure level densities in ${ }^{96} \mathrm{Mo}$ and ${ }^{56} \mathrm{Fe}$. These experiments are the first attempts to apply this technique to nuclei of importance to astrophysics and stockpile stewardship. The ${ }^{56} \mathrm{Fe}$ data will be the thesis topic for a graduate student (E. Tavukcu) from North Carolina State University stationed at LLNL. The ${ }^{56} \mathrm{Fe}$ experiment is of interest since recent nuclear structure calculations predict a sudden breakdown in the nuclear pairing field (typified by an increase in the level density) in this nucleus [Koo97]. Results from these experiments will be presented at the International Nuclear Physics Conference in July 2001.

Figure 3: The CACTUS array at the University of Oslo

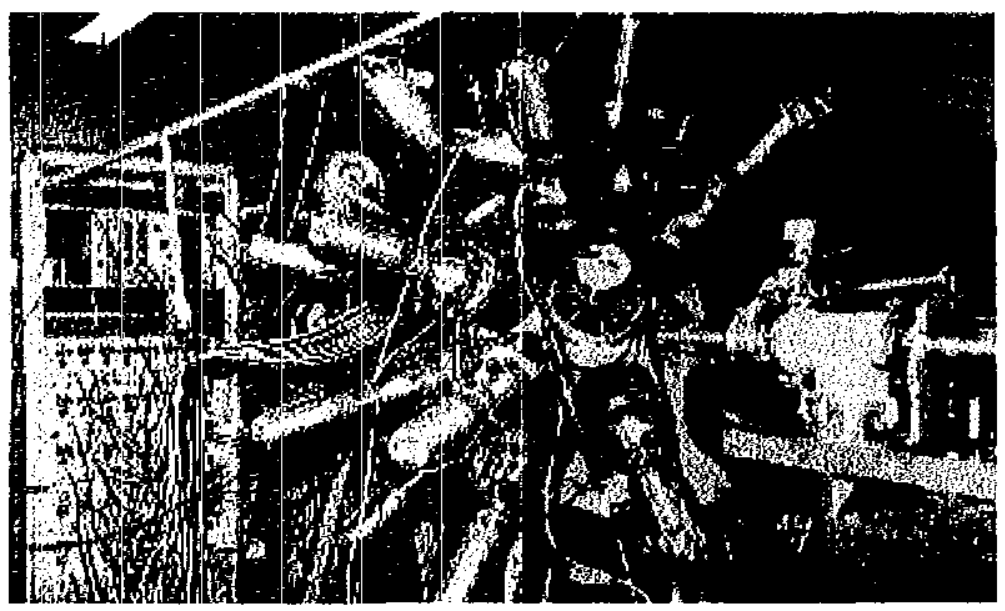


Figure 4: Data from the Oslo experiments showing the first generation $\gamma$-ray spectra at different excitation energy in the residual nucleus as determined by the energy of the outgoing $\alpha$-particle. The left panel is a two-dimensional plot of the first generation $\gamma$ rays observed ( $\mathrm{x}$-axis) as a function of excitation energy (y-axis). The arrows show the location of one-dimensional slices of the data showing $\gamma$-ray spectra corresponding to specific excitation energies.
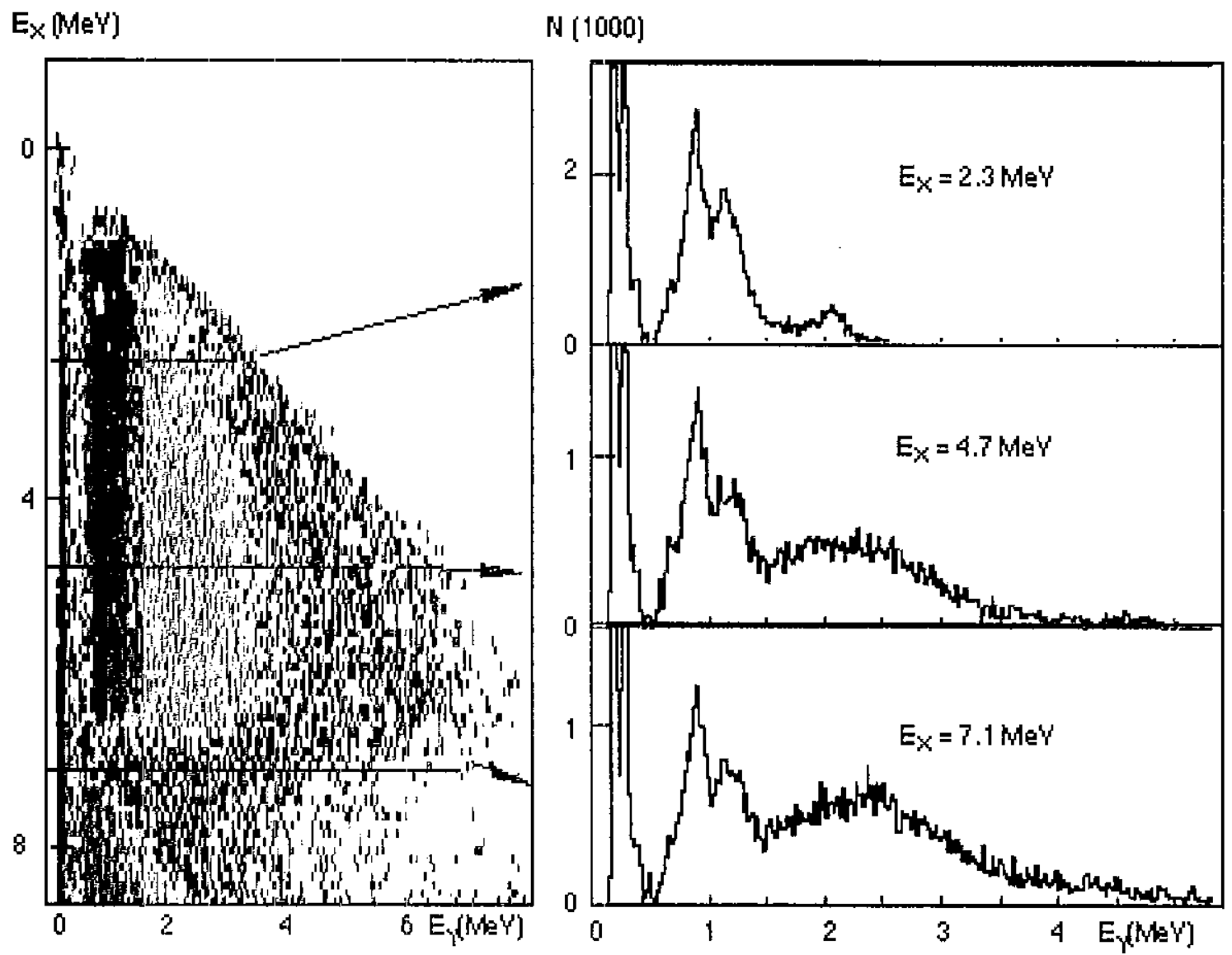

Level density measurements at the University of Oslo have been restricted to rare earth nuclei near $A=150-160$. However, nuclei in the mass 90 region play a critical role in both nuclear astrophysics (the formation of neutron deficient "p-process" nuclei), and radiochemistry $\left({ }^{89} \mathrm{Y}\right.$ and ${ }^{90} \mathrm{Zr}$ were commonly used radiochemical diagnostics). The purpose of the ${ }^{96} \mathrm{Mo}$ experiment was to perform the first level density measurement in this mass region. In addition, we have purchased target material to allow for future experiments in ${ }^{93}$ Mo that is located between the two "p-nuclei" ${ }^{92} \mathrm{Mo}$ and ${ }^{94} \mathrm{Mo}$. Dr. Andreas Schiller, a new post-doctoral researcher from the University of Oslo, is analyzing the ${ }^{96} \mathrm{Mo}$ data. 


\section{3) The Yale experiment}

In the course of the "normal" analysis of the ${ }^{97} \mathrm{Mo}$ Oslo data Dr. Schiller has identified a compelling structure in the ${ }^{97}$ Mo data corresponding to a highly coherent nuclear state at an unusually high energy $\left(\mathrm{E}_{\mathrm{x}}=5.3 \mathrm{MeV}\right)$. The high density of states in a nucleus at this excitation energy usually causes such states to be highly mixed over a wide range of energies. The existence of this state challenges conventional theories of nuclear structure. A follow-up experiment was run at Yale University in the September 2001 measured the energy of the outgoing $\alpha$-particle following the ${ }^{97} \mathrm{Mo}\left({ }^{3} \mathrm{He}, \alpha\right)^{96} \mathrm{Mo}$ transfer reaction using the Yale split-pole spectrometer. This experiment confirmed the existence of the state and located 2 similar states in neighboring Molybdenum isotopes. Figure 5 below shows the state in $\alpha$-particle spectrum from the Yale experiment.

T.F. Wang from the Analytical Nuclear Chemistry Division at LLNL is spearheading this effort. A second experiment is planned for Yale in late February 2001 which will determine which of the neighboring Molybdenum isotopes exhibit the similar structure. The experiment will be a collaborative effort between LLNL and the nuclear astrophysics group at Yale under the leadership of Prof. Peter Parker.

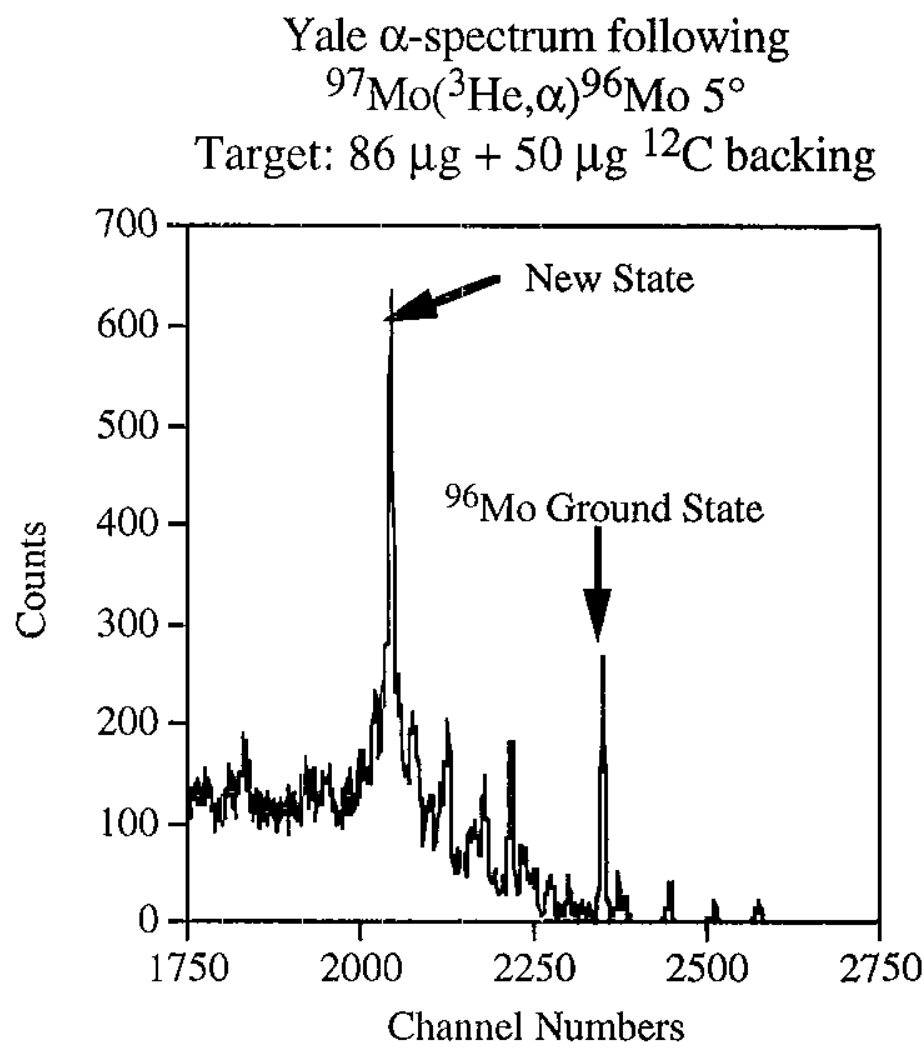

Figure 5: $\alpha$-particle spectrum from the Yale transfer experiment showing the new coherent state at $\mathrm{E}_{\mathrm{x}}=5.3 \mathrm{MeV}$ taken at $5^{\circ}$ in the lab frame. 


\section{4) The Development of "Re-cyclotron" Radioactive Beams at LBNL}

Intense radioactive beams with a lifetime greater than approximately 12 hours can be produced using the "re-cyclotron" technique. In the first part of a recyclotron experiment a large population of radioactive nuclei are formed during an 8-24 hour production run using a light-ion reaction. In the second the radioactive nuclei are removed from the production target and accelerated through the cyclotron onto a target.

Two experiments were run at LBNL in 2001 to develop the re-cyclotron technique. The results from these experiments were presented at the Division of Nuclear Physics meeting of the American Physical Society in October 2001. In the first experiment the feasibility of using the ${ }^{74} \mathrm{Se}(\alpha, 2 \mathrm{n}){ }^{76} \mathrm{Kr}\left(\mathrm{t}_{1.2}=14\right.$ hours $)$ and the ${ }^{74} \mathrm{Se}(\alpha, \mathrm{pn}){ }^{76} \mathrm{Br}\left(\mathrm{t}_{1 / 2}=16.1\right.$ hours $)$ was validated. In the second one a new production target was irradiated using a high-intensity (8-10 $\mu$ Ampere) $\alpha$-particle beam and the radioactive Krypton and Bromine gases were removed from the target by controlled heating and trapped in a two-stage cryo-trap system. A highly enriched target of $>99 \%{ }^{74}$ Se was used to maximize the production the isotopes of interest. A third test run scheduled for February 2002 will attempt to inject these radioactive gases into the ECR ion source. LLNL has two approved recyclotron experiments planned for LBNL in 2002. Figure 6 below shows recyclotron beam candidates in a portion of the chart of nuclides in the $A=90$ mass region.

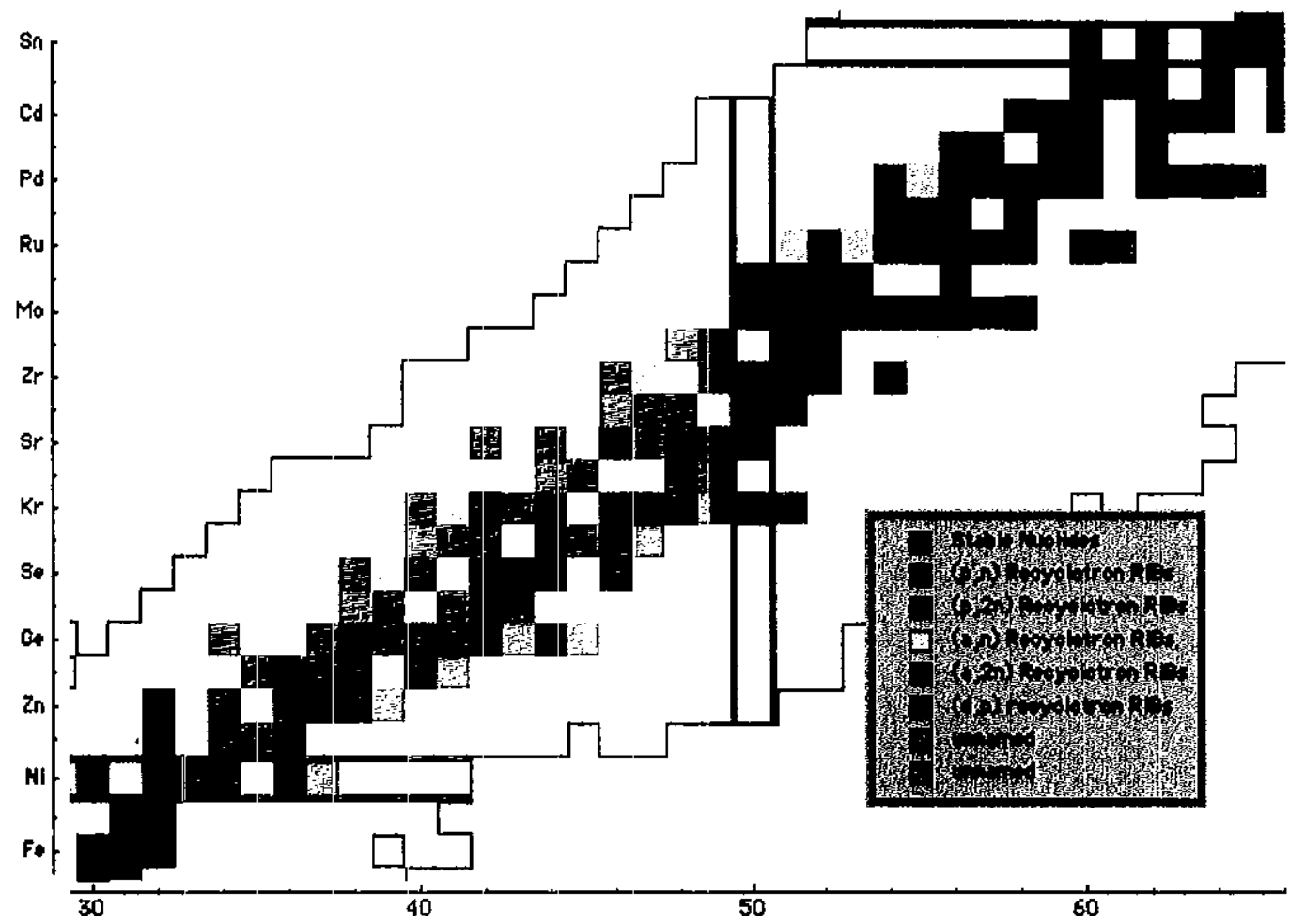

Figure 6: Recyclotron candidates in the $A=90$ region (colored squares). Stable nuclei are shown in black 


\section{5) The Development of a Highly Segmented Particle Detector (HSPD) for RIB Experiments}

Another major initiative started under this LDRD was the design of a new detector system to measure level densities, $\gamma$-ray strength functions and cross sections using inverse kinematics radioactive beams. The detector would have to be highly segmented in order to provide maximum particle energy resolution. Such a highly segmented particle detector (HSPD) would allow for Oslo-type experiments to be performed more quickly and with higher sensitivity using state-of-the-art $\gamma$-ray spectrometers in the U.S.

The HSPD is designed to be highly versatile. It includes a target chamber, electronics, and two different Silicon particle telescope arrays for use in forward and inverse kinematics experiments. The detectors designed for use in forward kinematics experiments, called the SiRi (Silicon Ring) array, are from collaborators at the University of Oslo. The detectors for use in inverse kinematics experiments are an "off-the-shelf" design from Micron semiconductor. An invited seminar describing the HSPD was given at LBNL in October 2001. The HSPD will enable level density and $\gamma$-ray strength function measurements reactions and cross section measurements using inverse kinematics RIBs. The first experiment using the array was approved by the LBNL PAC and will take place in April 2002 in conjuction with GAMMASPHERE at LBNL.

The coupling of the HSPD to GAMMASPHERE will provide a number of improvements over the Oslo set-up. Firstly, the HSPD has 20 times the efficiency of the particle detector array used at Oslo. Secondly, the GAMMASPHERE $\gamma$-ray efficiency for is 4-6 times higher than Oslo. However, the most striking improvement is the addition of the 110 high-resolution Ge detectors. These detectors will, for the first time, allow the measurement of the angular momentum dependence of the level density and the $\gamma$-ray strength function. This will be accomplished through observation of the statistical $\gamma$-ray spectrum coincident with discrete $\gamma$-rays observed in the Ge detectors that populate low-lying states of known angular momentum. The first "proof-of-principle" of the coupled GAMMASPHERE+HSPD will be a "normal" kinematics experiment using a stable target and beam. The proposal for this experiment was submitted in May.

In addition to the level density measurements, the HSPD will be used to measure $(D, p)$ cross sections by accelerating a re-cyclotron RIB onto a target of deuterated polyethylene. This cross section can be used as a surrogate for the $(n, \gamma)$ cross sections and to guide reaction model calculations. Future experiments using GAMMASPHERE or another high resolution $\gamma$-ray spectrometer coupled to the HSPD will therefore "kill two birds with one stone" and measure level densities/ $\gamma$-ray strength function and $(D, p)$ cross sections simultaneously.

The first of these coupled experiments will be proposed in the fall of 2001 . We will use the ${ }^{76} \mathrm{Kr}$ beam developed this year in inverse kinematics to measure: a) level densities and $\gamma$-ray strength functions in ${ }^{77} \mathrm{Kr}$ via the $\mathrm{d}\left({ }^{76} \mathrm{Kr}, \mathrm{p}\right){ }^{77} \mathrm{Kr}$ reaction, and $\left.\mathrm{b}\right)$ the ${ }^{76} \mathrm{Kr}(\mathrm{D}, \mathrm{p}){ }^{77} \mathrm{Kr}$ cross section. The second experiment, using a ${ }^{77} \mathrm{Br}$ recyclotron RIB was proposed and approved for running in late 2002. 


\section{6) The ANL Secondary Beam Experiment}

A technique that has successfully produced radioactive beams is the use of secondary beams. Secondary beams can be easily formed using a number of heavy-ion reactions at intensities suitable for cross section measurements. An example is the measurement of the coulomb excitation cross section on the $\mathrm{J}^{\pi}=4^{-}$isomer in ${ }^{78} \mathrm{Rb}$ formed via the ${ }^{24} \mathrm{Mg}\left({ }^{58} \mathrm{Ni}, 3 \mathrm{pn}\right)$ reaction using ATLAS (Argonne Tandem and Linear Accelerator System) coupled to the FMA (Fragment Mass Analyzer) at Argonne National Laboratory [Sch99]. The residue was separated using the FMA. Complete reconstruction of the event kinematics was also used to isolate $\gamma$-rays from the large background.

In February 2001 we collaborated with the Michigan State University Nuclear Physics group on a secondary beam experiment using ATLAS+FMA. In this experiment a radioactive beam of ${ }^{176} \mathrm{Os}$ was formed using the ${ }^{48} \mathrm{Ti}\left({ }^{132} \mathrm{Xe}, 4 \mathrm{n}\right)$ reaction at $640 \mathrm{MeV}$. The ${ }^{176} \mathrm{Os}$ beam was then used to initiate Coulomb excitation reactions on a Nickel target at the focal plane of the FMA. Figure 7 below shows the FMA at ANL:

The goal of this experiment was to measure to measure the deformation of the low-lying states in ${ }^{176}$ Os. This nucleus is of interest due to its "transitional" nuclear structure, which has aspects of both rotational and vibrational degrees of freedom. The results of this work have been presented at the American Physical Society meeting in April 2001.

Figure 7: The Fragment Mass Analyzer at Argonne National Laboratory

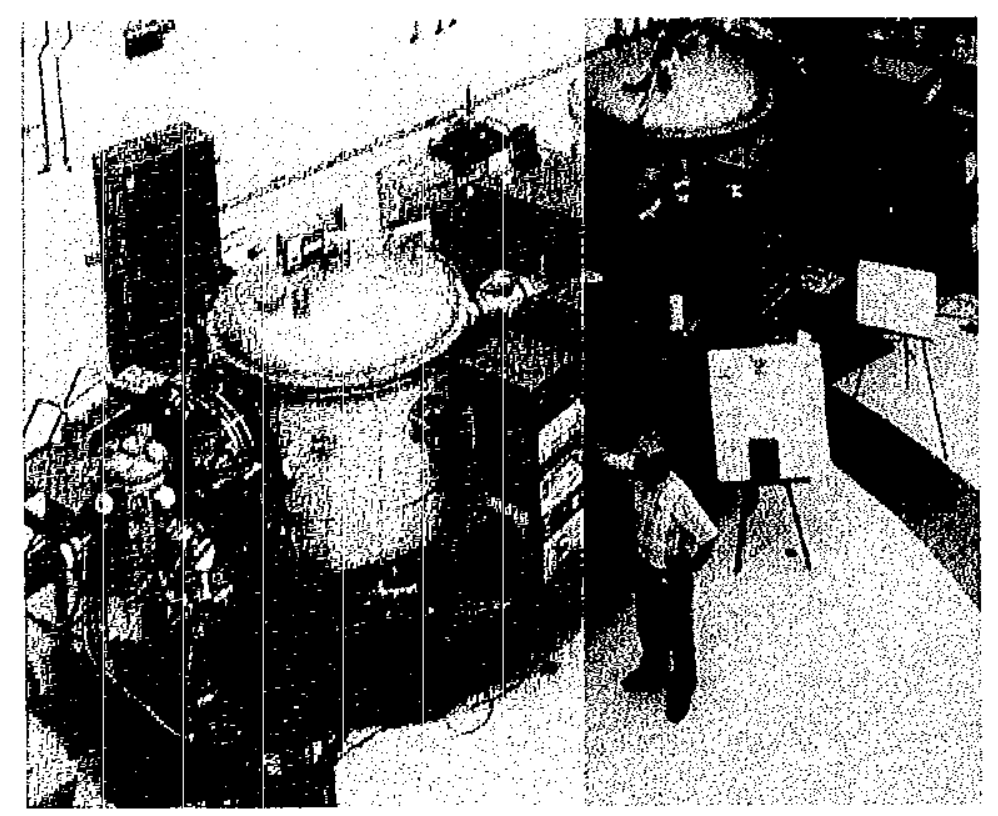




\section{7) RIBs and Stockpile Stewardship Workshop at LLNL}

A classified workshop was organized at LLNL in November of 2000 in order to get a better understanding of how RIA and RIB experiments could better serves the needs of stockpile stewardship community. Representatives from both A- and B-divisions were present at the meeting, as well as staff from Los Alamos National Laboratory. Experiments using RIA to support stockpile stewardship needs were discussed and prioritized lists for reaction cross sections were drawn up. The program for this meeting is shown below.

\section{SBSS Applications for RIA, 8 November 2000, LLNL}

$\begin{array}{lll}\frac{\text { Start Time }}{8: 30} & \begin{array}{l}\text { Presenter } \\ \text { Dennis Slaughter }\end{array} & \frac{\text { Title }}{\text { RIA facility description }} \\ \begin{array}{ll}\text { Session 1: } \\ 9: 00\end{array} & \begin{array}{ll}\text { Nuclear Physics Experiments } \\ \text { Mark Stoyer }\end{array} & \begin{array}{l}\text { Stockpile Stewardship } \\ \text { Radiochemistry and Radioactive } \\ \text { Beams }\end{array} \\ 9: 30 & \text { Lee Bernstein } & \begin{array}{l}\text { Cross Section Measurements for } \\ \text { Stockpile Stewardship using RIA }\end{array} \\ \text { 10:00 } & \text { Jim Hall/ } & \text { An Intense Neutron Source for RIA } \\ 10: 20 & \begin{array}{l}\text { Brian Rusnak } \\ \text { discussion }\end{array} & \end{array}$

Session 2: Nuclear Modeling

11:15 W. Erich Ormand

11:45 Rob Hoffman

Discussion over lunch

Session 3: Nuclear Theory

13:30 Mohammed Mustafa

14:00 Mort Weiss

14:30 Discussion

Session 4: Design issues

14:30 Steve Libby

14:50 Bob Little

15:10 Steve Becker

16:00 Discussion

Session 5: Other issues

16:30 Mike Kreisler

16:45 Discussion

17:30 All
Nuclear Structure and Reaction

Modeling

Radiochemistry Modeling Issues

Nuclear Physics Issues in

A-program

SBSS Questions!

T.B.D.

Fission Fragments and Fission

Products in SBSS: Suggested

Studies with a Rare Isotope

Accelerator

SBSS R\&D Overview

N-division RIA Program

Close/summary/action items 


\section{3) Summary of Deliverables and Future Plans}

In fiscal year 2002 the funding for this project was moved from LDRD to the Physical Data Research Program (PDRP). This transfer reflects an understanding that RIB experiments offer significant opportunities for improving the interpretation of archival radiochemical data and for maintaining a supply of forefront scientific staff to the lab. The establishment of an ongoing programmatic commitment has increased the ability of the program to take on longer-term RIB projects.

The full accomplishments of this LDRD will become more evident as results from the HSPD and recyclotron RIB experiments begin in 2002 and 2003. However, at the time that this report was being prepared the following deliverables could be attributed to it:

- The thesis project of Emel Tavukcu, a North Carolina State University graduate student stationed at LBNL who is analyzing the ${ }^{56} \mathrm{Fe}$ Oslo data.

- Two refereed publications in progress based on two Oslo data sets.

- Three invited seminars at the University of California - Berkeley, Lawrence Berkeley National Laboratory, and Duke University.

- Two presentations at the Division of Nuclear Physics Meeting of the American Physical Society Mini-symposium on Radioactive Beam facilities.

- The recruitment of 2 new post-doctorals: Drs. Andreas Schiller (1/01) and Jeffrey Cooper (10/01) with a third post-doctoral likely to start in 7/02 (Jason Burke).

In addition to publications and recruitment/retention of scientific staff there are other tangible benefits linked to this proposal's success including:

- Growth of our existing ( $\$ 300 \mathrm{k}$ in FY00) DOE/Office of Science/Nuclear Physics funding. DOE/OS/NP has indicated that increased funding is contingent upon our move into RIA-oriented activities.

- New stockpile stewardship program activities. These tools will provide nuclear data needed to improve radiochemical reaction networks.

The years ahead hold great promise for the use of RIB experiments. We are looking forward to a productive near and long-term future.

\section{References:}

[Sau97] T. Sauter and F. Kappeler, Phys. Rev. C55, 3127 (1997).

[Woo94] S.E.Woosley, J.R. Wilson, G.J. Matthews, R.D. Hoffman and B.S. Meyer, The Astrophysical Journal, 433, 229-246 (1994).

[Hof96] R.D. Hoffman, S.E. Woosley, G.M. Fuller and B.S. Meyer, The Astrophysical Journal, 460, 478-488 (1996).

[Pre84] R.J. Prestwood, K.W. Thomas, D.R. Nethaway and N.L. Smith, Phys. Rev. C29, 805 (1984).

[Bao00] Z. Y. Bao, H. Beer, F. Kappeler, F. Voss, and K. Wisshak, At. Data Nucl. Data Tables 76 70-154 (2000).

[Ber97] L.A. Bernstein et al., Phys. Rev. C 57 R2799 (1998). UCRL-ID-130176

[Gar00a] P.E. Garrett et al., Phys. Rev. C, submitted. UCRL-JC-136968. 
[Net99] D.R. Nethaway and M. Mustafa. Measured Data Used in the Watusi Cross-Section Sets. UCRL-ID-133269 (1999) and E.A. Henry, private communications.

[Hau52] W. Hauser and H. Feshbach Phys, Rev. 87, 366 (1952).

[Gut87] M.Guttormsen, T.Ramsoy and J.Rekstad, Nucl. Instrum. A255 518, (1987).

[Tve96] T.S.Tveter, L.Bergholt, M.Guttormsen, E.Melby and J.Rekstad. Phys.Rev.Lett. 77, 2404 (1996).

[Miz99] S. Mizuno et al., Journal of Nuclear Science and Technology, 36, 493 (1999).

[You01] W. Younes, J.A. Becker, L.A. Bernstein, P.E. Garrett, C.A. McGrath, D.P. McNabb, R.O. Nelson, G.D. Johns, W.S. Wilburn, D.M. Drake Phys. Rev. C64, 054613-1 (2001).

[Eng93] T.R. England and B.F. Rider, LANL report LA-UR-94-3106 (1993).

[Voi01] A. Voinov, M. Guttormsen, E. Melby, J. Rekstad, A. Schiller, S. Siem gamma-ray strength function and pygmy resonance in rare earth nuclei Phys. Rev. C 63, 044313 (2001).

[Koo97] S.E. Koonin, D.J. Dean, and K. Langanke, Phys. Reports 278 (1997) 1, and references therein. 